Pereira, Dora; Perales, María-Jesús \& Bakieva, Margarita (2016). Análisis de tendencias en las investigaciones realizadas a partir de los datos del Proyecto PISA. RELIEVE, 22(1), art. M10. DOI: http://dx.doi.org/10.7203/relieve.22.1.8248

Revista ELectrónica de Investigación y EValuación Educativa
RELIEVE

ISSN: 1134-4032
e-Journal of Educational Research,

Assessment and Evaluation

\title{
Análisis de tendencias en las investigaciones realizadas a partir de los datos del Proyecto PISA
}

\author{
Trends analysis in the investigations realised from the data of the PISA Project
}

\author{
Pereira, Dora ${ }^{(1)}$; Perales, María-Jesús ${ }^{(2)}$ \& Bakieva, Margarita ${ }^{(2)}$
}

(1) Piaget- Institute (Portugal) (2) Universidad de Valencia (España)

\begin{abstract}
This study is part of a broader research that aims to design a model of evaluation of educational systems, based in social cohesion. The starting point is located in previous research about the context variables; it is cared more frequently a theoretical justification and metric quality of output variables, leaving aside the variables that can help interpret the results. In our case, we conducted a review of the scientific literature, based in empirical papers that use PISA database and published between 2000 and 2012. The methodology is structured in two phases: identification and selection of documents and further analysis of these documents. The analysis of documents consists of identifying certain indicators in order to explore. Some results suggest that researchers in the field of education underuse PISA databases.
\end{abstract}

Reception Date

2016 April 3

Approval Date

2016 June 14

Publication Date:

2016 June 14

\section{Keywords:}

OCDE; PISA; educational systems; empirical research; educational research

\section{Resumen}

Este estudio es parte de una investigación más amplia que pretende diseñar un modelo de análisis de evaluaciones de sistemas educativos que permita valorar su aportación para la cohesión social. El punto de partida se sitúa en las investigaciones previas que analizan el papel de las variables de contexto, de entrada y de proceso en las evaluaciones de sistemas educativos, que frecuentemente cuidan mucho más la justificación teórica y la calidad métrica de las variables de producto que estas otras, que finalmente ayudan a interpretar los resultados. En este caso realizamos revisión de los trabajos científico-académicos del corte empírico, publicados entre los años 2000 y 2012, que utilizan bases de datos PISA. La metodología se estructura en dos fases: identificación y selección de los documentos para formar una base y posterior análisis de cada uno de los documentos con el fin de extraer indicadores que nos permitieran realizar un análisis exploratorio. Algunos resultados hacen pensar que las bases de datos PISA están infrautilizadas, sobre todo por los investigadores en el campo de educación.

Fecha de recepción 3 Abril 2016

Fecha de aprobación 14 Junio 2016

Fecha de publicación 14 Junio 2016

\section{Palabras clave:}

OCDE; PISA; sistemas educativos; investigación empírica; investigación educativa

PISA (Programa Internacional de Evaluación de Estudiantes, por sus siglas en inglés) es ampliamente conocido por cualquier persona interesada en el estado de la educación de su país o a nivel internacional. Como es sabido, el Proyecto PISA analiza la educación en países que representan un $90 \%$ de economía mundial; monitorea sus sistemas educativos a través de la evaluación de competencias básicas a la edad de 15 años, con el objetivo de ofrecer información relevante para establecer propuestas políticas y prácticas para llegar a una educación efectiva (Ozmusul \& Atanur, 2013).

El PISA se desarrolla en ciclos de 3 años, y evalúa la competencia lingüística, científica o matemática, y más recientemente evalúa también la capacidad de resolución de problemas y la competencia financiera. En cada oleada PISA se elige uno de los tres 
dominios evaluados como dominio principal (Tabla 1). Esta coherencia diacrónica en la evaluación de niveles de competencias y no de conocimientos curriculares específicos permite que las conclusiones de PISA sean un arma poderosa de influencia sobre las políticas educativas de muchos países (Bottani, 2006).

Tabla 1- Áreas de Estudio PISA por oleadas

\begin{tabular}{ccc}
\hline Oleada & Dominio Principal & Dominios Secundarios \\
\hline 2000 & Lectura & Matemática y Ciencias \\
2003 & Matemática & Lectura y Ciencias \\
2006 & Ciencias & Lectura y Matemática \\
2009 & Lectura & Matemática y Ciencias \\
2012 & Matemática & Lectura y Ciencias \\
2015 & Ciencias & Lectura y Matemática \\
\hline
\end{tabular}

El organismo que proporciona infraestructura, materiales y especialistas para realización del estudio PISA es la OCDE (Organización para la Cooperación y el Desarrollo Económico), siempre que las naciones participantes o las instituciones educativas que la utilizan paguen una cuota de participación. Ofrece las bases de datos del PISA abierta y gratuitamente a los investigadores a través de su página web oficial $^{1}$. Estas bases de datos ofrecen un abundante volumen de información sobre alumnado y centros de los países estudiados, extraída con gran rigor estadístico a nivel muestral, y ofreciendo amplia información sobre la elaboración de indicadores, lo cual permite incluso comparar diferentes países (Cordero, Crespo \& Pedraja, 2013).

La propia OCDE ofrece una gran cantidad de informes con explicaciones de los datos y las inferencias sobre la población, así como manuales de análisis metodológico y sobre las teorías en las que se sustenta el diseño de las pruebas tanto contextuales, como de rendimiento. Efectivamente, en el proyecto PISA se evidencia una gran evolución, y con el paso del tiempo intenta ofrecer más información que la que se aparece inicialmente en los propios informes. Así, a partir del año 2002 se empiezan a generar Informes Secundarios, con autoría de expertos, unos vinculados a la OCDE y otros investigadores

\footnotetext{
${ }^{1}$ https://www.oecd.org/pisa/pisaproducts
}

independientes. Estos informes explican la relación entre las diferentes variables analizadas y los resultados de evaluación. Actualmente ya podemos contar con un listado bastante amplio de informes secundarios, documentos explicativos de PISA, en diferentes idiomas, en cuanto a descripción de metodología de evaluación y análisis, profundizan en los resultados, y ofrecen sugerencias y recomendaciones de política educativa a partir del análisis de los resultados ${ }^{2}$.

Paralelamente, los informes de la OCDE se utilizan como base para justificar políticas de alto impacto y la toma de decisiones sobre políticas educativas. Para Acevedo (2007) las evaluaciones internacionales están dirigidas especialmente a los gestores y administradores de la educación -policy oriented studies- con el objetivo de permitirles tomar decisiones para el impulso y la orientación de las reformas de la enseñanza. Estos estudios, como PISA, proveen pruebas empíricas que ofrecen soporte para consideraciones teóricas e ideológicas.

Las pruebas de PISA están diseñadas para conocer las habilidades y competencias de los estudiantes para analizar y resolver problemas, tratando de ofrecer, de forma longitudinal, un perfil de las capacidades de los estudiantes de todos los países donde se aplica. Aunque PISA

\footnotetext{
${ }^{2}$ http://www.mecd.gob.es/inee/publicaciones/estudiosinternacionales.html
} 
reconoce en sus fundamentos su pretensión de incidir en las políticas educativas, lo cierto es que también reconoce no estar ligado al currículum ni a planes de estudios específicos (Gallardo-Gil et al., 2010). Se entiende, más que como un simple dispositivo de evaluación comparativa internacional de los resultados escolares, como un complejo proceso de revisión y reflexión para la regulación política trasnacional.

Para Carvalho (2009) PISA concierta inquisición y medición, es un "tablero de promoción de acuerdos sobre cuáles son las prácticas y las políticas educativas que los gobiernos nacionales admiten someter a escrutinio externo, armando de forma regular a los políticos de datos y análisis elaborados con base en modelos construidos sobre convenciones establecidas por expertos" (Carvalho, 2009, p. 1017).

Hay quien opina que los indicadores de PISA se centran en la comparación de los efectos y consecuencias entre sistemas educativos, más que en sus resultados, en un dinamismo de mutua prestación de cuentas. Carvalho (2009) destaca que la audiencia principal del programa son los decisores políticos, de forma que la información es presentada en los informes según sus necesidades y peticiones. Se trata de fabricar conocimiento para la política. Es pues un punto de origen y a la vez de llegada de decisiones políticas.

PISA produce conocimiento sobre el conocimiento. Los informes efectuados para tomar decisiones políticas deben ser la materia prima y primaria para otros análisis secundarios realizados por especialistas de distintas áreas del conocimiento, que trabajan las evidencias sobre los resultados de los alumnos en matemática, ciencia o lengua respeto a otras variables.

Aun siendo informes deficientemente difundidos, e insuficientemente leídos y comprendidos, los resultados más visibles, como los rankings, tienen un gran impacto social y un alto seguimiento en prensa (Batista,
2016; Jornet, 2013, 2016), siendo utilizados incluso para justificar reformas en las legislaciones educativas.

Las críticas a los informes tipo PISA son numerosas. Éstas se refieren tanto a los aspectos técnico-metodológicos y validez de los estudios (Gorur, 2014), como a la exactitud de medida de equidad del PISA (Rutkowski \& Rutkowski, 2013), o a aspectos metodológicos de comparación entre países (Torney-Purta, 2013) y otros (Cordero, Crespo \& Pedraja, 2013).

De hecho, de acuerdo con Jornet (2013) existen diferentes asignaturas pendientes en las evaluaciones a gran escala, y entre ellas destaca la relacionada con los usos de la información recogida en ellas. En el caso concreto de PISA la crítica más habitual es la falta de capacidad explicativa de los estudios evaluativos junto con el escaso aprovechamiento que realiza la comunidad científica de las bases de datos evaluativos.

Esto se relaciona con el hecho de que la cantidad de estudios empíricos realizados a partir de los datos ofrecidos desde las bases de PISA parezca escasa. Está claro que PISA no es un proyecto de investigación, sin embargo los datos generados pueden resultar de gran interés para los investigadores (Turner, 2006). Aunque se trata de bases de datos amplias y sólidas, con un gran potencial para la investigación empírica, parece que son escasamente utilizadas por investigadores de entidades académicas y/o independientes. Esta aparente falta de compromiso de los investigadores con el aprovechamiento $\mathrm{y}$ explotación de estos datos, pese a provenir de un estudio con un gran impacto social, ha sido el centro de la investigación que aquí se presenta, revisando las características de los estudios empíricos que sí han sido publicados con ese perfil.

\section{Objetivos}

Este estudio es parte de una investigación más amplia que pretende diseñar un modelo de evaluación de sistemas educativos que permita valorar su aportación para la Cohesión Social. 
El punto de partida en esa investigación son estudios previos sobre el papel de las variables de contexto, de entrada y de proceso en las evaluaciones de sistemas educativos, que frecuentemente cuidan mucho más la justificación teórica y la calidad métrica de las variables de producto (rendimiento, evaluación de competencias) que estas otras variables que, al final, serán las que ayuden en la interpretación de resultados (esta línea de trabajo fue desarrollada en el Proyecto MAVACO EDU2009-13485 financiado por el Ministerio de Ciencia e Innovación). Las líneas generales del actual proyecto, denominado SECS/EVALNEC, se describen en Jornet (2012). Brevemente, el Modelo de evaluación para Cohesión Social de Jornet propone una serie de instrumentos diseñados con el propósito de interpretar los resultados de los estudiantes en función de otros elementos de contexto (De la Orden \& Jornet, 2012) como pueden ser la metodología docente, valor subjetivo de educación, colegialidad docente y la visión de justicia social subjetiva (Jornet, 2012). Esta serie de elementos son indicadores de calidad de un sistema educativo, basada en la cohesión social e inclusividad.

De acuerdo con las líneas marcadas por el proyecto subyacente, en este estudio realizamos una revisión inicial de los trabajos científico-académicos del corte empírico, publicados entre los años 2000 y 2012, que utilizan bases de datos PISA. Dichos trabajos deben estar centrados en analizar la vinculación entre las variables consideradas de contexto y los resultados escolares evaluados por PISA. Los objetivos de esta investigación, por lo tanto, se han centrado en:

- Presentación del proceso de revisión bibliográfica realizado, sus criterios y sus resultados;

- Presentación de los estudios empíricos que cumplen los criterios de inclusión en el estudio;

- Caracterización del contenido de los documentos, en función del país/países estudiados, el nivel de análisis, la metodología estadística utilizada y el tipo de recomendaciones aportadas.

\section{Metodología}

La metodología de este estudio se estructura en dos fases. La primera se centró en la creación de la base documental objeto de estudio y la identificación de los documentosbase de estudio y la segunda en el análisis de documentos, según protocolos de trabajo que permiten identificar información cuantificable y/o cualitativa.

\section{Fase I.}

La primera fase, tal y como se mencionó, se centró en la identificación y selección de los documentos de análisis. La metodología de revisión documental siguió los pasos sugeridos por Bisquerra (2014), y tuvo como objetivo identificar los estudios empíricos realizados desde instituciones académicas y de investigación ajenas a la OCDE y publicados entre 2000 y 2012, que utilizaran las bases de datos ofrecidas por PISA para investigar sobre la vinculación de variables llamadas de contexto (variables de contexto, entrada y proceso) y los resultados educativos medidos por PISA. La base documental de estudio se construye con artículos publicados en revistas científicas fundamentalmente, así como con ponencias y/o comunicaciones (papers), working-papers, y capítulos de ebook, que presenten estudios empíricos realizados sobre las bases de datos PISA que estén accesibles a partir de las bases de datos de la Universidad de Valencia ${ }^{3}$.

Somos conscientes que, aunque abordemos la búsqueda de forma sistemática en una amplia gama de bases de documentación científica, con seguridad siempre quedarán trabajos no localizados que no serán, por tanto, parte del estudio.

Para minimizar esta problemática, la aproximación documental se ha estructurado en función de los criterios utilizados para

\footnotetext{
${ }^{3}$ Bases de datos a las que se ha suscrito la Universitat de València: trobes.uv.es
} 
seleccionar las bases de documentación, que han sido los siguientes:

- amplitud y prestigio de las bases consultadas;

- especialización o relación con la temática tratada (proyecto PISA, informes y/o proyectos de evaluación de sistemas educativos, estudios sobre políticas y organización de la educación, explicación del rendimiento, logro o aprendizaje, estudios transculturales, estudios comparados, estudios educativos basados en aproximaciones con pruebas a gran escala).

Asimismo, completada la búsqueda inicial, se han realizado búsquedas de comprobación a partir de buscadores genéricos, como Google y Google Académico, con el fin de poder rastrear otros trabajos que no estuvieran indexados en las bases analizadas.

La búsqueda se realizó, siempre que la base de datos lo permitió restringir, para artículos escritos en una de las cuatro lenguas: inglés, francés, castellano y portugués.

En las búsquedas se ha utilizado un conjunto de palabras-clave muy reducido, con el fin de poder identificar el mayor número de trabajos posible. De este modo, como palabras-clave se han considerado: PISA, OCDE, rendimiento y variables de contexto.

La finalidad era identificar los artículos publicados en revistas científicas que presentaban estudios empíricos que hubieran trabajado con las bases de datos del Proyecto PISA. Por este motivo, las palabras-clave de inicio de búsqueda fueron PISA y OCDE. No obstante, si bien son términos que actualmente están presentes de forma muy frecuente en la literatura educativa en general, y de evaluación en particular, también es cierto que la mayor parte de documentos que pueden devolverse en una búsqueda a partir de ellos hacen referencia a los informes (general o por países) o a reflexiones relacionadas con los usos de los resultados del Proyecto, su utilidad, la controversia social que provoca, etc. Estudios empíricos que se hayan realizado sobre sus bases de datos son sustancialmente menos frecuentes. Las palabras-clave 3 y 4 (rendimiento y variables de contexto) pretendían acotar ligeramente los resultados, eliminando sólo los trabajos de reflexión teórica, dejando en los resultados de la búsqueda los estudios empíricos. Por este motivo, tampoco se pueden considerar términos que restrinjan de manera importante los resultados ofrecidos en las búsquedas.

La selección final se basó en la revisión directa de los trabajos encontrados, de forma que se pudiera comprobar que realmente cumplían el objetivo central: ser estudios empíricos realizados sobre bases de datos provenientes del Proyecto PISA. Los artículos que no correspondían a este tipo de estudios mencionado, se descartaron. Por este motivo, constituyó un trabajo muy laborioso, al tener que acceder directamente a las fuentes originales (los artículos científicos, papers y working papers, capítulos). Sin embargo, considerando que la calidad de la investigación depende en gran medida de la adecuación de la información disponible, entendimos que era un trabajo fundamental dirigido a apoyar la validez de la investigación

De este modo, se dispone de un banco de documentos integrado por 116 trabajos (artículos científicos, papers, work-papers y capítulos de ebook).

En la tabla 2 se presentan diferentes soluciones de búsqueda en diversas bases documentales. Las búsquedas se circunscribieron a los años de 2000 hasta el 2012. 
Tabla 2 - Parámetros de búsqueda

\begin{tabular}{lccc}
\hline & \multicolumn{3}{c}{ Parámetros de búsqueda booleana } \\
\cline { 2 - 4 } Fuente & $\begin{array}{c}\text { (PISA) and } \\
\text { (OCDE) }\end{array}$ & $\begin{array}{c}\text { (PISA) and (OCDE) and } \\
\text { (Variables de Contexto) or } \\
\text { (Variáveis de Contexto) }\end{array}$ & $\begin{array}{c}\text { (PISA) and (OCDE) and } \\
\text { (Variables predictoras) or } \\
\text { (Variáveis predictoras) }\end{array}$ \\
\hline Google & 59600 & 5040 & 12800 \\
Google Académico & 31500 & 4050 & 123 \\
troves.uv.es & 20 & 0 & 0 \\
SpringerLink & 32 & 0 & 0 \\
Sciencedirect.com & 30 & 0 & 0 \\
ProQuest.com & 942 & 22 & 0 \\
persee.fr & 12 & 0 & 0 \\
Wiley Online Library & 11 & 1 & 0 \\
Scopus & 158 & 0 & 0 \\
\hline
\end{tabular}

\section{Fase II}

Una vez terminada la Fase I y constituida la base de documentación, se procedió a la siguiente Fase II que se centró en el análisis de cada uno de los documentos, con el fin de extraer indicadores cuantitativos (datos) y cualitativos (argumentos), que nos permitieran realizar un análisis exploratorio basado en la revisión del material científico a partir del criterio de varios jueces.

Después de reunir todo el material que cumple con las características marcadas hemos realizado análisis descriptivos utilizando el SPSS versión 22 con licencia de la Universitat de València.

\section{Resultados}

Este apartado describe los resultados que han sido obtenidos en cada una de las dos fases del estudio.

Fase I. Creación de la base documental objeto de estudio: identificación de documentos-base de estudio.

La primera fase permitió identificar un gran número de documentos publicados con investigaciones realizadas a partir de las bases de datos de PISA. Estos documentos fueron revisados para comprobar el cumplimiento de los criterios de inclusión/ exclusión en la investigación (ordenados por su nivel de relevancia):

1. Estudios empíricos.

2. Realizados por instituciones académicas o de investigación ajenas a la OCDE.

3. Que revisaran la vinculación entre las llamadas variables de contexto y los resultados escolares medidos por PISA, siendo ésta considerada variable de producto.

En la primera selección se identificaron 248 trabajos científicos. Casi la mitad de ellos cumplían efectivamente con los criterios de inclusión de la revisión bibliográfica planteada, pero otros tantos, tras el análisis detallado del documento, fueron excluidos por diferentes motivos. En la Tabla 3 se presenta la cantidad de documentos excluidos según los distintos criterios. 


\section{Tabla 3 - Criterios de exclusión}

\begin{tabular}{lc}
\hline \multicolumn{1}{c}{ Motivo de Exclusión } & Frecuencia \\
\hline No es accesible en abierto o solo resumen (abstract) & 62 \\
No es un trabajo empírico & 21 \\
No tiene variables PISA como variable de Producto & 13 \\
Otra lengua que no el castellano, portugués, inglés o francés & 2 \\
Informe Oficial/OCDE o Nacional (Ministerio, Instituto de Estadística o de Evaluación) & 20 \\
Solo en formato papel & 14 \\
No es un paper/ working paper & 1 \\
\hline
\end{tabular}

El primer criterio de exclusión de los documentos encontrados se refiere a la accesibilidad. Después, fueron eliminados los documentos que correspondían a informes oficiales de la OCDE, fuese a nivel orgánico o de las agencias nacionales, pues el objetivo de la investigación se refiere a investigaciones realizadas desde instituciones académicas o de investigación ajenas a la OCDE. Finalmente, el tercer gran motivo de exclusión se refiere al contenido del documento: pese a que estos 248 estudios pre-seleccionados hacían referencia a una investigación aparentemente empírica, un análisis más detenido de su contenido reveló que la investigación aportaba datos, pero en sí misma no había hecho un análisis empírico de los mismos, o que no utilizaba los resultados de PISA como variable de producto.
Así, tras la revisión de contenido, la base documental queda constituida por 116 documentos científicos con indicios de calidad científica, basados en estudios empíricos realizados a partir de bases de datos PISA.

Fase II. Análisis de documentos, según protocolos de trabajo que permiten identificar información cuantificable y/o cualitativa.

La mayoría de los estudios revisados han sido publicados como artículo científico, según se presenta en la Tabla 4. Otro formato frecuente es de working papers, documentos de trabajo conjunto, desarrollado durante algunas jornadas académico-científicas o investigadoras, siendo los capítulos de libros en e-books en abierto los menos habituales.

Tabla 4 - Tipo de trabajo

\begin{tabular}{lcccccc}
\hline & \multicolumn{7}{c}{ Ola de PISA analizada } \\
\cline { 2 - 7 } Tipo & $\mathrm{N}^{*}$ & 2000 & 2003 & 2006 & 2009 & Total \\
\hline Artículos & 72 & 24 & 21 & 34 & 2 & 81 \\
W/P & 37 & 9 & 15 & 16 & 6 & 46 \\
E-books/ Capítulos del libro & 7 & 0 & 1 & 5 & 2 & 8 \\
\hline \multicolumn{1}{c}{ Total } & 116 & 33 & 37 & 55 & 10 & 135 \\
\hline
\end{tabular}

Nota: El total de trabajos de esta tabla (Total, columna final) no es igual al número de trabajos analizados en el estudio ( $\mathrm{N}$, primera columna), porque el mismo trabajo puede tratar varias oleadas de PISA.

Una primera descripción de estos estudios se centra en las oleadas PISA estudiadas. El diseño de PISA, como se ha indicado, se organiza en Oleadas sucesivas que profundizan en diferentes áreas de conocimiento y que a lo largo del tiempo permiten ir completando todo el universo considerado. La Tabla 5 muestra cuántos de los documentos revisados (presentados por año de publicación) analizan cada una de las Oleadas. Se puede observar un lógico tiempo de carencia entre la realización de las evaluaciones por parte de la OCDE y la publicación de los primeros estudios independientes; de hecho, al cabo de cuatro o cinco años se inicia el periodo de máxima 
productividad respecto a la oleada considerada. Esto se debe en primer lugar al tiempo que toma PISA en publicar las bases de datos, después al tiempo requerido para plantear y desarrollar la investigación independiente, $\mathrm{y}$ finalmente al tiempo para publicar.

Tabla 5 - Año de publicación por oleada PISA y total de trabajos científicos

\begin{tabular}{|c|c|c|c|c|c|c|c|c|c|c|}
\hline & \multicolumn{2}{|c|}{ Total $(\mathrm{n}=116)$} & \multicolumn{2}{|c|}{2000} & \multicolumn{2}{|c|}{2003} & \multicolumn{2}{|c|}{2006} & \multicolumn{2}{|c|}{2009} \\
\hline & $\mathrm{F}$ & $\%$ & $\mathrm{~F}$ & $\%$ & $\mathrm{~F}$ & $\%$ & $\mathrm{~F}$ & $\%$ & $F$ & $\%$ \\
\hline 2001 & 2 & 1,72 & 1 & 3,03 & & & & & 1 & 10,00 \\
\hline 2003 & 2 & 1,72 & 2 & 6,06 & & & & & & \\
\hline 2004 & 3 & 2,59 & 3 & 9,09 & & & & & & \\
\hline 2005 & 7 & 6,03 & 6 & 18,18 & 1 & 2,70 & & & & \\
\hline 2006 & 5 & 4,31 & 2 & 6,06 & 4 & 10,81 & & & & \\
\hline 2007 & 11 & 9,48 & 6 & 18,18 & 6 & 16,22 & & & & \\
\hline 2008 & 10 & 8,62 & 3 & 9,09 & 7 & 18,92 & 2 & 3,64 & & \\
\hline 2009 & 10 & 8,62 & 2 & 6,06 & 5 & 13,50 & 7 & 12,73 & & \\
\hline 2010 & 28 & 24,14 & 5 & 15,15 & 7 & 18,92 & 21 & 38,18 & & \\
\hline 2011 & 28 & 24,14 & 2 & 6,06 & 7 & 18,92 & 17 & 30,91 & 6 & 60,00 \\
\hline 2012 & 10 & 8,62 & 1 & 3,03 & & & 8 & 14,55 & 3 & 30,00 \\
\hline Total & 116 & 100,00 & 33 & 100,00 & 37 & 100,00 & 55 & 100,00 & 10 & \\
\hline
\end{tabular}

Nota: Los porcentajes se calculan por columna, siendo el cien por cien el total de trabajos científicos por cada oleada PISA y la columna Total es la suma de todos los trabajos científicos de las cuatro oleadas. Un mismo trabajo científico puede agrupar dos o más oleadas PISA, por lo que suma por fila no sería igual al total de trabajos publicados en ese año.

Si se analiza la adscripción de los investigadores, se observa que hay una gran cantidad de estudios realizados colaborativamente desde varias instituciones, lo cual explica que el total de países instituciones formantes excede con mucho el número de 116. Además, se evidencia que hay una gran preponderancia de investigadores europeos, según se describe en la Tabla 6 .

Tabla 6 - Localización de las instituciones a las que se adscriben los y las investigadores

\begin{tabular}{lccccc}
\hline \multicolumn{1}{c}{ Región } & Total & 2000 & 2003 & 2006 & 2009 \\
\hline Europa & 113 & 32 & 39 & 49 & 10 \\
Australia y Nueva Zelanda & 6 & 2 & 0 & 2 & 2 \\
Asia & 14 & 5 & 5 & 8 & 0 \\
América del Norte & 13 & 5 & 1 & 6 & 1 \\
América Central y del Sur & 2 & 0 & 1 & 1 & 0 \\
Iberoamérica & 34 & 2 & 10 & 20 & 6 \\
Internacional & 1 & 0 & 1 & 0 & 0 \\
\hline
\end{tabular}

Nota: Las casillas contienen las frecuencias de países en las que se localizan las instituciones que dan cobertura a los trabajos científicos. Estos países se agrupan por regiones de correspondencia tanto económico-geográfica, como lingüística. Así, la región Iberoamericana agrupa países de Europa y Latinoamérica de habla hispana o portuguesa.

En algunos casos el número total de la fila supera a la suma por cada oleada de PISA; esto se debe a que algunos estudios se realizan en colaboración entre instituciones de diferentes países.

La categoría “Internacional” se refiere al Banco Mundial.

La Tabla 6 muestra claramente que en la mayor parte de los estudios están implicados científicos de instituciones europeas (113 en total). Esto puede indicar una mayor sensibilidad en Europa respecto a estudios internacionales como PISA (frente a Estados 
Unidos, por ejemplo, que va posiblemente a mantener como referente más significativo sus estudios nacionales), y también una mayor tendencia y/o facilidades para desarrollar estudios colaborativos que impliquen a entidades de diferentes países.

Los países de América Central y del Sur están representados en los documentos estudiados solamente por instituciones de dos países, en el Centro de Investigación Avanzada en Educación (CIAE) y en el Departamento de Economía de la Universidad de Chile, y la Escuela de Administración y Finanzas e Instituto Tecnológico (EAFIT), de Colombia.

Respecto a esta infra representación, Martínez Rizo (2006, p. 154) explica que “como la mayoría de los países de desarrollo intermedio o bajo, los países de América Latina no tienen una tradición importante en este campo". El autor subraya, además, la importancia de que la participación de estos países “sea lo más activa posible” (p. 166).

En los 116 trabajos científicos seleccionados participan investigadores de 34 instituciones de la región de Iberoamérica. Pero, como se ha indicado, sólo 2 son de América Central y del Sur, siendo 28 de instituciones españolas y otros 4 de instituciones portuguesas.

Finalmente, América del Norte alberga 13 investigaciones en total, los países de Oriente
14 investigaciones, Australia y Nueva Zelanda 6.

El idioma de publicación podría parecer un criterio de exclusión de investigaciones realizadas en zonas de Asia, por ejemplo. Sin embargo, al incluir los cuatro idiomas más frecuentes en el mundo (inglés, francés, portugués y español), se ha abordado como un criterio inclusivo. Sin embargo, la selección de bases de datos consultadas sí puede actuar como criterio excluyente pues puede suceder que recojan de forma deficitaria la publicación de países externos a Europa y Norteamérica.

En la mayor parte de las regiones, como en los datos totales, el número de investigaciones se incrementa con las sucesivas oleadas PISA (siendo especialmente evidente en Europa y en Iberoamérica). Esto puede ser un reflejo del interés creciente por el proyecto, a la vista de su impacto político y social, y también de la mayor accesibilidad real de las bases de datos. Sin embargo, en algunas regiones con menos frecuencia de estudios esta tendencia se rompe en el estudio de PISA 2003, siendo difícil encontrar una primera explicación para ello.

En la Tabla 7 se ofrece el recuento de los ámbitos de las revistas en las que han sido publicadas las investigaciones. Se observa una clara tendencia hacía revistas del corte económico, lo cual condiciona el carácter de las investigaciones $\mathrm{y}$ el área de los investigadores que publican en ellas.

Tabla 7 - Ámbito de la revista

\begin{tabular}{|c|c|c|c|c|c|c|}
\hline Año del Artículo & Educación & Economía & CC.SS y Psicolog.* & Ingeniería & Sociología & Total \\
\hline 2001 & & 1 & 1 & & & 2 \\
\hline 2003 & & 2 & & & & 2 \\
\hline 2004 & & 3 & & & & 3 \\
\hline 2005 & 4 & 2 & 1 & & & 7 \\
\hline 2006 & 3 & 2 & & & & 5 \\
\hline 2007 & 1 & 8 & 2 & & & 11 \\
\hline 2008 & 2 & 6 & & 1 & 1 & 10 \\
\hline 2009 & 3 & 6 & 1 & & & 10 \\
\hline 2010 & 12 & 13 & 3 & & & 28 \\
\hline 2011 & 8 & 17 & 3 & & & 28 \\
\hline 2012 & 3 & 4 & 3 & & & 10 \\
\hline Todos & 36 & 64 & 14 & 1 & 1 & 116 \\
\hline
\end{tabular}

Nota: * Incluye Psicología y CC. del Comportamiento. 
Aunque la OCDE es claramente un organismo económico, el Proyecto PISA es un estudio educativo. Pese a ello, la Tabla 7 muestra que más del $50 \%$ de los documentos analizados se encontraban en revistas o publicaciones del ámbito de la Economía, muestra que la Educación, siendo el segundo grupo, se quedaba en el $25 \%$. Esto puede mostrar que los investigadores que pertenecemos al ámbito de educación reaccionamos tarde y con poco impacto, por lo menos a nivel de cantidad de estudios. Hay pocos investigadores del área de educación que se dedican a utilizar los datos de PISA en sus investigaciones.

Presentados los investigadores, es relevante analizar las regiones estudiadas en los diferentes documentos considerados. Así, en la Tabla 9 podemos observar la clasificación de estudios científicos en función de la región estudiada. Los datos muestran claramente que la mayor parte de los estudios investigados se centran en países de Europa.

Tabla 9 - Región foco del estudio

\begin{tabular}{lccccc}
\hline Región & Total $(\mathrm{n}=116)$ & $2000(\mathrm{n}=33)$ & $2003(\mathrm{n}=37)$ & $2006(\mathrm{n}=55)$ & $2009(\mathrm{n}=10)$ \\
\hline América Central y del Sur & 45 & 19 & 10 & 18 & 3 \\
América del Norte & 49 & 19 & 16 & 19 & 2 \\
Australia y Oceanía & 61 & 20 & 20 & 23 & 2 \\
Asia & 104 & 24 & 41 & 50 & 5 \\
\hline Europa & 733 & 239 & 226 & 301 & 31 \\
Iberoamérica & 127 & 39 & 35 & 57 & 9 \\
\hline
\end{tabular}

Nota: n-número de estudios científicos correspondientes a la oleada PISA y al Total (primera columna). Las casillas contienen el número de países foco del estudio en cada caso (total o por oleada de PISA). La suma por fila no coincide con el valor en la columna Total ya que algunos estudios revisan múltiples oleadas de PISA.

La suma por columnas tampoco coincide con el $\mathrm{N}$ total (por oleada y total global) dado que muchos estudios incluyen múltiples países foco en sus estudios.

Iberoamérica agrupa países de Europa y Latinoamérica de habla hispano-portuguesa.

Tal y como se puede ver en la Tabla 9, los estudios se focalizan básicamente en Europa, siendo España, Alemania, Italia, Finlandia y Reino Unido los países más frecuentemente utilizados como elemento central de estudio. Un estudio de publicaciones más recientes mostraría, probablemente, una mayor preferencia por países sistemáticamente valorados positivamente en PISA, como Finlandia. En el periodo analizado, es presumible que se prioricen en mayor medida criterios como el análisis del país de procedencia de los investigadores, o la comparación con regiones significativas.

Iberoamérica es, a mucha distancia, la siguiente región más estudiada, con 127 países que aparecen como países foco en los 116 documentos. Sin embargo, analizando de qué países se trata, se observa una gran presencia de España y, en menor medida, Portugal, de forma que la frecuencia de menciones de países de América Central y del Sur considerados foco es de 45. Entre ellos, destaca México, como el más atendido, considerado foco en 25 ocasiones, lo cual es coherente con la participación de este país en PISA, junto con Brasil, desde su primera edición. Esto confirma la importancia para estos países de participar en estudios como PISA, que visibiliza sus sistemas educativos, en la línea antes planteada por Martínez Rizo (2006).

En cuanto a los países asiáticos Corea y Japón son los más analizados, y destaca la ausencia de China que decidió no participar en el Proyecto PISA (excepto regiones específicas como Hong Kong y Macao).

En la revisión de contenido de la investigación realizada se han considerado dos elementos descriptivos fundamentales. En 
primer lugar, el nivel de análisis, y después el tipo de análisis realizado en la investigación.

La Tabla 10 ofrece los datos sobre el nivel del análisis de las investigaciones empíricas. Los Informes PISA, y las bases de datos que ofrecen, se estructuran en función de diferentes niveles de análisis, permitiendo distintos tipos de agrupaciones de los datos, que se vinculan con distintos objetivos de investigación. Aunque el estudio no permite sacar conclusiones a nivel de alumnado ni de centros concretos, pues no está diseñado para ello, las bases ofrecen la información a nivel de sujetos y agrupada por centros. Esto permitirá hacer análisis vinculados a distintas variables individuales sobre las que se haya recogido información (como características demográficas, sociales, económicas, o de trayectoria escolar, entre otras), o sobre características de los centros escolares (como la titularidad o el tamaño).

Los siguientes niveles de análisis considerados son el país y la región, considerada ésta como una agrupación de países por criterio geográfico.

A primera vista se puede observar que la mayoría de los trabajos científicos utilizan estudios a nivel de alumno o grupo de alumnos, seguido de número de investigaciones que estudian variables a nivel de centro escolar o tipos de centros, seguido de variables a nivel país o por comunidades del país y finalmente a nivel de la región, que puede agrupar varios países.

Tabla 10 - Nivel de análisis

\begin{tabular}{lcccccccccc}
\hline Nivel de & \multicolumn{2}{c}{ Total $(\mathrm{n}=116)$} & \multicolumn{2}{c}{$2000(\mathrm{n}=33)$} & \multicolumn{2}{c}{$2003(\mathrm{n}=37)$} & \multicolumn{2}{c}{$2006(\mathrm{n}=55)$} & \multicolumn{2}{c}{$2009(\mathrm{n}=10)$} \\
\cline { 2 - 12 } Análisis & $\mathrm{F}$ & $\%$ & $\mathrm{~F}$ & $\%$ & $\mathrm{~F}$ & $\%$ & $\mathrm{~F}$ & $\%$ & $\mathrm{~F}$ & $\%$ \\
\hline Alumno & 115 & 99,14 & 0 & 0,00 & 0 & 0,00 & 54 & 98,18 & 0 & 0,00 \\
Centro & 99 & 85,34 & 28 & 84,85 & 35 & 94,59 & 44 & 80,00 & 9 & 90,00 \\
País & 34 & 29,31 & 7 & 21,21 & 12 & 32,43 & 17 & 30,91 & 5 & 50,00 \\
Región & 27 & 23,28 & 10 & 30,30 & 10 & 27,03 & 11 & 20,00 & 1 & 10,00 \\
\hline
\end{tabular}

Nota: Nivel de análisis Alumno - por grupos de alumnos; Centro - por tipos de centros escolares; País - por comunidades, provincias, zonas o regiones internas de un país; Región - zonas que agrupan varios países. El porcentaje es respecto al número de trabajos total por ola de PISA y en total de todos los trabajos analizados.

Desde la Tabla 10 podemos observar que la tendencia al análisis a nivel de distintas variables es diferente según la ola del PISA. En los estudios que utilizan datos del PISA 2000, 2003 y 2009 no existen estudios que contrastan variables a nivel alumno o grupos de alumnos. Sin embargo, los estudios basados en el PISA 2006 en un 98,18\% de los casos utilizan este tipo de variables o indicadores.

Evidentemente, los niveles de análisis no son mutuamente excluyentes entre sí. En este sentido, se observa que con el paso del tiempo los análisis realizados a las sucesivas oleadas combinan niveles de análisis distintos. Así, si en las investigaciones revisadas sobre la Oleada 2000 los análisis se realizan sobre todo a nivel de centro, y la escasa duplicidad de frecuencias indica que sólo se hicieron a ese nivel, en los análisis realizados sobre la Oleada 2006 se observa que los análisis se efectúan simultáneamente a nivel de sujetos y de centro, combinando informaciones.

Para la descripción de los análisis estadísticos utilizados en cada caso se han planteado cinco categorías, que tampoco son mutuamente excluyentes entre sí:

- Análisis descriptivos univariados o bivariados, que ofrecen una presentación global de las variables consideradas objeto de estudio.

- Contraste de hipótesis para comprobar diferencias o similitudes entre grupos establecidos por distintas características, y la influencia de unas u otras variables. Entre las investigaciones que realizan 
contraste de hipótesis, se ha comprobado si valoran o no el tamaño del efecto. Esto se considera especialmente relevante como precaución de investigación dado que el gran tamaño de las muestras disponibles en PISA puede influir en los resultados que aparecen en los contrastes de hipótesis.

- Análisis multivariados de diferente tipo. Agrupan varias variables para estudiar la influencia combinada entre ellas. Dependiendo de la investigación, pueden ser Análisis Mutivariables de Varianza (MANOVA), Regresión Múltiple, Análisis Factorial, Análisis
Discriminante, Análisis de Conglomerados o Clústeres, Escalamiento Multidimensional y/o Modelos Causales.

- Análisis multinivel. Forman parte de un grupo de modelos de análisis estadístico que permiten tratan los conjuntos de datos anidados dentro de una población con estructura jerárquica, entendiendo que los distintos niveles de jerarquía son niveles de análisis (Amador \& LópezGonzález, 2007). En la Figura 1 se puede observar un ejemplo de estructuración de los datos en dos niveles.

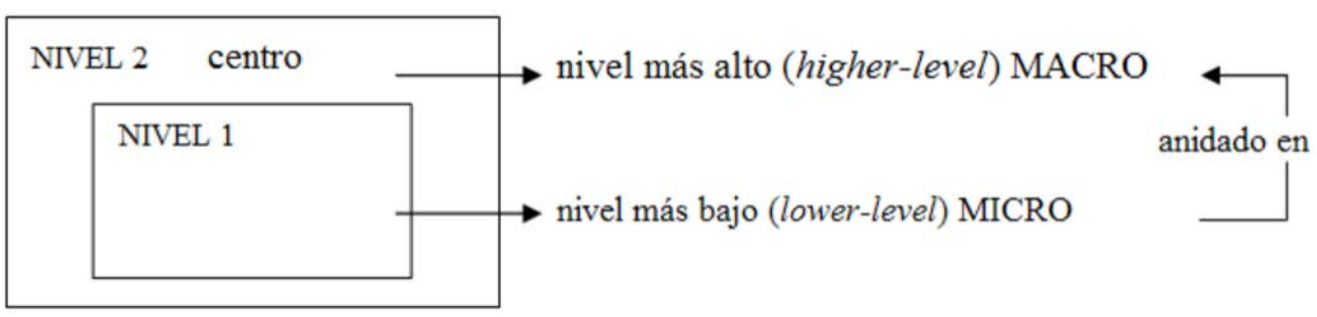

Figura 1. Estructura de los datos en los análisis multinivel.

Fuente: Amador y López-González (2007)

La Tabla 11 ofrece datos agrupados sobre el tipo de análisis utilizado en las investigaciones. Grosso modo, el nivel avanzado de los procedimientos estadísticos no llega a ser avanzado en la mayoría de los casos. De hecho, en línea con el estudio de Pey-Yan y Yi-Chen (2015), encontramos que los efectos de tamaño y diseño, ajustes esenciales para analizar datos de estudios a gran escala, se detectan en menos de la mitad de casos. Sin embargo, las pruebas de significancia estadística pueden ser insuficientes en los estudios de esta envergadura, donde la magnitud del efecto observado es fundamental. Los procedimientos estadísticos del tamaño del efecto están destinados para cuantificar ese efecto y son necesarios en las poblaciones de estudios tan amplias (Coe \& Merino, 2003). Su ausencia relativa en estos análisis es relevante pues puede implicar que se puedan cuestionar algunos de los resultados, y puede ser indicador de falta de tradición en el análisis de grandes muestras desde entidades ajenas a los grandes organismos internacionales.

Tabla 11 - Tipo de análisis utilizados en el estudio

\begin{tabular}{lcccccccccc}
\hline & \multicolumn{2}{c}{ Total $(\mathrm{n}=116)$} & \multicolumn{2}{c}{$2000(\mathrm{n}=33)$} & \multicolumn{2}{c}{$2003(\mathrm{n}=37)$} & \multicolumn{2}{c}{$2006(\mathrm{n}=55)$} & \multicolumn{2}{c}{$2009(\mathrm{n}=10)$} \\
\cline { 2 - 11 } & $\mathrm{F}$ & $\%$ & $\mathrm{~F}$ & $\%$ & $\mathrm{~F}$ & $\%$ & $\mathrm{~F}$ & $\%$ & $\mathrm{~F}$ & $\%$ \\
\hline Descriptivo & 65 & 56,03 & 17 & 51,52 & 25 & 67,57 & 31 & 56,36 & 6 & 60,00 \\
Contraste Hipótesis & 80 & 68,97 & 27 & 81,82 & 30 & 81,08 & 35 & 63,64 & 4 & 40,00 \\
-- Tamaño efecto & 38 & 32,76 & 12 & 36,36 & 17 & 45,95 & 12 & 21,82 & 3 & 30,00 \\
Multivariado & 82 & 70,69 & 23 & 69,70 & 26 & 70,27 & 35 & 63,64 & 9 & 90,00 \\
Multinivel & 62 & 53,45 & 17 & 51,52 & 17 & 45,95 & 26 & 47,27 & 7 & 70,00 \\
\hline
\end{tabular}

Nota: El porcentaje es respecto al número de artículos total por ola de PISA y en total de los trabajos analizados. Algunos trabajos utilizan varios niveles de análisis en el trascurso de la investigación. 
Los análisis multivariados son los más frecuentes en las investigaciones revisadas (más de un $70 \%$ del total de documentos los utilizan siendo claramente más frecuentes en los análisis realizados sobre la Oleada 2009), pues permiten una aproximación muy pertinente en el fenómeno educativo, que por definición es muy complejo. Los análisis multivariados, al abordar de forma global la influencia y las relaciones de interdependencia de distintas variables, desde diferentes aproximaciones, reflejan de una forma adecuada la complejidad de la educación.
Una de las consecuencias más relevantes de los estudios PISA son las recomendaciones y reflexiones que se pueden extraer. Por ello, la última categoría de análisis respecto a los 116 estudios revisados va a ser ésta.

En la Tabla 12 se puede observar la tendencia de recomendaciones que se realizan en los artículos, agrupados por año de publicación. Claramente, el número de recomendaciones va en aumento a lo largo de los años, mostrando un crecimiento bastante alto.

Tabla 12 - Tipo de recomendaciones realizadas en el estudio

\begin{tabular}{|c|c|c|c|c|c|c|c|c|c|c|}
\hline \multirow[t]{2}{*}{$\begin{array}{c}\text { Año de } \\
\text { publicación }\end{array}$} & \multicolumn{2}{|c|}{ Total* } & \multicolumn{2}{|c|}{$\begin{array}{l}\text { Recomendacione } \\
\text { s sobre Política } \\
\text { Educativa }\end{array}$} & \multicolumn{2}{|c|}{$\begin{array}{c}\text { Recomendaciones } \\
\text { Didácticas }\end{array}$} & \multicolumn{2}{|c|}{$\begin{array}{c}\text { Recomendaciones } \\
\text { sobre } \\
\text { Metodología } \\
\text { PISA } \\
\end{array}$} & \multicolumn{2}{|c|}{$\begin{array}{l}\text { Recomendaciones } \\
\text { para Futuras } \\
\text { Investigaciones }\end{array}$} \\
\hline & $\mathrm{F}$ & $\%$ & $\mathrm{~F}$ & $\%$ & $\mathrm{~F}$ & $\%$ & $\mathrm{~F}$ & $\%$ & $\mathrm{~F}$ & $\%$ \\
\hline 2001 & 2 & 1,72 & 1 & 0,86 & 0 & & 1 & 0,86 & 1 & 0,86 \\
\hline 2003 & 2 & 1,72 & 2 & 1,72 & 0 & & 0 & & 2 & 1,72 \\
\hline 2004 & 3 & 2,59 & 2 & 1,72 & 2 & 1,72 & 2 & 1,72 & 2 & 1,72 \\
\hline 2005 & 7 & 6,03 & 6 & 5,17 & 2 & 1,72 & 2 & 1,72 & 2 & 1,72 \\
\hline 2006 & 5 & 4,31 & 5 & 4,31 & 3 & 2,59 & 1 & 0,86 & 2 & 1,72 \\
\hline 2007 & 11 & 9,48 & 10 & 8,62 & 3 & 2,59 & 4 & 3,45 & 5 & 4,31 \\
\hline 2008 & 10 & 8,62 & 7 & 6,03 & 6 & 5,17 & 6 & 5,17 & 4 & 3,45 \\
\hline 2009 & 10 & 8,62 & 5 & 4,31 & 4 & 3,45 & 2 & 1,72 & 2 & 1,72 \\
\hline 2010 & 28 & 24,14 & 25 & 21,55 & 10 & 8,62 & 11 & 9,48 & 18 & 15,52 \\
\hline 2011 & 28 & 24,14 & 25 & 21,55 & 12 & 10,34 & 2 & 1,72 & 13 & 11,21 \\
\hline 2012 & 10 & 8,62 & 6 & 5,17 & 2 & 1,72 & 2 & 1,72 & 6 & 5,17 \\
\hline Todos & 116 & 100,00 & 94 & 81,03 & 44 & 37,93 & 33 & 28,45 & 57 & 49,14 \\
\hline
\end{tabular}

Nota: La última columna contiene número total de artículos por cada año. No tiene que ser igual a la suma de la fila correspondiente, porque en el mismo artículo pueden darse recomendaciones de diferentes tipos. El porcentaje se calcula a partir del número total de artículos ( $\mathrm{n}=116)$.

Al año siguiente de la primera aplicación PISA ya se publican dos estudios. En ellos, se realizan recomendaciones sobre política educativa, metodología de PISA (tipo evaluativa o análisis) y recomendaciones para investigaciones futuras.

Un $81 \%$ de los documentos revisados ofrecen recomendaciones sobre políticas educativas, siendo mucho menor el porcentaje de documentos que ofrecen revisiones sobre estrategias didácticas, entendiendo que es un ámbito mucho más específico. Con todo resulta significativo señalar que, aunque sólo un $25 \%$ de los estudios se han publicado en revisas del ámbito educativo, los investigadores han encontrado conclusiones suficientes para hacer recomendaciones a un ámbito que no es inicialmente el suyo. Este potencial interdisciplinar de los estudios de evaluación de sistemas debe ser apoyado y cuidado, para que realmente sea una forma constructiva de abordar el fenómeno de la educación, pero sin permitir que la voz de pedagogos/as y especialistas en educación sea obviada.

También es relevante señalar que casi un $50 \%$ de los estudios ofrece recomendaciones para futuras investigaciones. Frecuentemente, terminar una investigación es el momento idóneo para plantear otra, tanto por los 
interrogantes que se abren a partir de los resultados encontrados, como por las pautas metodológicas de análisis que se han podido contrastar o refutar. En este sentido, es imprescindible recalcar el carácter acumulativo de las líneas de investigación y la necesidad de fomentar redes de investigación que permitan profundizar colaborativamente sobre los tópicos más relevantes.

Finalmente, también destaca que casi un $30 \%$ de los estudios revisados reflexionen sobre la propia metodología PISA. La dificultad técnica del estudio, y su gran impacto político y social (que además ha ido creciendo con el tiempo) hacen necesario que el proceso de revisión sobre la propia metodología implicada en las diferentes fases del estudio (desde la identificación del constructo y el universo de medida, hasta el análisis y difusión de resultados) sea constante, y esté alimentado por reflexiones e ideas procedentes de los equipos investigadores de PISA pero también desde aportaciones externas. En este sentido, estas reflexiones de carácter metodológico deben ser consideradas por el propio equipo PISA como un tesoro sobre el que pensar cuidadosamente.

Tabla 13 - Tipo de recomendaciones por ámbito de la revista

\begin{tabular}{lccccccccc}
\hline & \multicolumn{8}{c}{ Tipo de recomendaciones } & \\
\cline { 2 - 8 } & \multicolumn{2}{c}{ Política Educativa } & \multicolumn{2}{c}{ Didácticas } & \multicolumn{3}{c}{ Metodología PISA } & \multicolumn{2}{c}{ Futuras } \\
Ámbestigaciones & Total \\
& $\mathrm{N}$ & $\%$ & $\mathrm{~N}$ & $\%$ & $\mathrm{~N}$ & $\%$ & $\mathrm{~N}$ & $\%$ & $(100 \%)$ \\
\hline Educación & 32 & 88,89 & 22 & 61,11 & 11 & 30,56 & 22 & 61,11 & 36 \\
Economía & 54 & 84,38 & 17 & 26,56 & 15 & 23,44 & 28 & 43,75 & 64 \\
CC.SS* & 8 & 57,14 & 5 & 29,41 & 6 & 42,86 & 5 & 29,41 & 14 \\
Ingeniería & & & & & 1 & 100,00 & 1 & 100,00 & 1 \\
Sociología & & & & & & & 1 & 100,00 & 1 \\
\hline
\end{tabular}

Nota: CC. SS. Incluyen las CC. de Comportamiento y Psicología

En cuanto a las diferentes tipos de recomendaciones, realizadas por los investigadores de cada uno de los trabajos analizados, en la Tabla 13 podemos observar cómo se distribuyen en función de ámbito de la revista o publicación.

Sólo hay una revista del ámbito de sociología, y otra de ingeniería, y las dos centran sus recomendaciones en comentarios para futuras investigaciones, o en cuestiones sobre la metodología de PISA. Si vamos a las más frecuentes, las publicaciones del ámbito de economía y de educación, la situación cambia. Comparativamente, los documentos publicados en el ámbito de la educación son más prolijos en recomendaciones de todo tipo. Las recomendaciones sobre política educativa son las más frecuentes, estando presentes en casi un $90 \%$ de los estudios publicados en el ámbito de la educación, pero, curiosamente, también en más de un $80 \%$ de los del ámbito económico. Las recomendaciones didácticas sí quedan en mayor medida en los estudios publicados en el ámbito de la educación (un $61.11 \%$, frente a un $26.56 \%$ de los estudios publicados en el ámbito económico). Ciertamente, las grandes políticas educativas son muy interdisciplinares, y el ámbito económico tiene su propia perspectiva al respecto. En el ámbito del aula, de la forma de dar las clases, de la didáctica, las recomendaciones sí son mucho más frecuentes en las revistas de educación, y eso a pesar de que PISA puede parecer un estudio alejado de las aulas. Tanto en las publicaciones del ámbito económico como en las del ámbito educativo, las recomendaciones sobre la propia metodología PISA son las menos frecuentes, pero están presentes en alrededor de un $25 \%$ de los estudios. La complejidad propia de PISA hace difícil hacer aportaciones sobre metodología, pero éstas pueden ser especialmente relevantes cuando se formulan desde la independencia y 
Pereira, Dora; Perales, María-Jesús \& Bakieva, Margarita (2016). Análisis de tendencias en las investigaciones realizadas a partir de los datos del Proyecto PISA. RELIEVE, 22(1), art. M10. DOI: http://dx.doi.org/10.7203/relieve.22.1.8248

externalidad de los investigadores, el interés específico por ámbitos y cuestiones concretas, y la experiencia y el conocimiento sobre las evaluaciones de sistemas educativos.

\section{Conclusiones}

El estudio que se presenta parte de la conciencia de la calidad y exhaustividad de las bases de datos ofrecidas por PISA, y de la sospecha de que están infrautilizadas. Y los resultados obtenidos vienen a confirmar esta sospecha, esbozando algunas posibles causas, con el objetivo de ofrecer pistas para dinamizar su uso.

Si, según Carvalho (2009), la principal virtud de PISA es fabricar conocimiento para la política, se puede considerar fundamental que también desde entidades ajenas a la OCDE pero interesadas en la investigación sobre educación se pueda colaborar a ese proceso, desde otras perspectivas y prioridades. Y para ello, la oportunidad que brindan las bases de datos ofrecidas por PISA no debe ser dejada de lado.

La OCDE ha mejorado esta disponibilidad de los datos. Las bases de datos cada vez son de más fácil acceso, y existen documentos del propio equipo PISA con indicaciones sobre cómo realizar los análisis de forma adecuada. Pese a ello, las bases siguen siendo complejas, y los datos muy prolijos, tanto en cantidad de constructos $\mathrm{y}$ variables, como en cantidad de sujetos. Y esto dificulta el manejo de las bases. Su mayor ventaja es también su mayor dificultad.

El estudio cuyas conclusiones presentamos se basa en un proceso de búsqueda documental. Se buscaban documentos disponibles, en inglés, francés, portugués o español, publicados desde 2001, que presentaran resultados de estudios empíricos desarrollados a partir de las bases de datos de PISA, donde los resultados PISA fueran variable de producto, a contrastar o analizar en función de otras variables de contexto. Las conclusiones que se presentan parten de la calidad de esta búsqueda. Del mismo modo, se puede aportar que las bases de datos consultadas probablemente identifican mejor publicaciones del ámbito europeo y americano que de contextos como el asiático, que probablemente está sesgadamente infrarrepresentado. Las limitaciones del estudio, por tanto, se vinculan con las palabras clave utilizadas y las bases consultadas, así como con la disponibilidad de los estudios, puesto que se ha partido de la base de la Universitat de València.

La revisión muestra que los estudios analizados se realizan sobre todo desde instituciones del ámbito europeo, y se focalizan también en países europeos. Se publican sobre todo en el ámbito económico, y en bastante menor medida educativo, tras un periodo habitual de carencia de unos cinco años desde la publicación de los datos.

La preponderancia de Europa como contexto investigador e investigado revela un particular interés de esta región por este tipo de estudios. PISA resulta para Europa un estudio contextualmente relevante, que aporta información interesante para sus propias políticas educativas, en un contexto habituado a la colaboración y el acuerdo para diseñar políticas conjuntas. Del mismo modo, las instituciones de investigación europeas disponen de medios y personas con formación e instrumentos para poder analizar este tipo de bases de datos, y habituadas a dinámicas de investigación colaborativas. En otras regiones del mundo, estas situaciones no se dan del mismo modo y, por un menor interés y/o por menor disponibilidad de posibilidades, se implican menos en realizar $\mathrm{y}$ publicar estudios empíricos de este tipo.

En cuanto a la metodología de análisis utilizada, el contraste de hipótesis y los estudios multivariados son los más frecuentes. Paulatinamente se utilizan estrategias metodológicas que respetan en mejor medida la complejidad del fenómeno educativo. Sin embargo, parece necesaria una reflexión metodológica sobre las estrategias utilizadas y la forma de hacerlo. Así, la evidencia de que sólo en un tercio de los estudios que realizan contraste de hipótesis se controla el tamaño del efecto, siendo algo fundamental en 
Pereira, Dora; Perales, María-Jesús \& Bakieva, Margarita (2016). Análisis de tendencias en las investigaciones realizadas a partir de los datos del Proyecto PISA. RELIEVE, 22(1), art. M10. DOI: http://dx.doi.org/10.7203/relieve.22.1.8248

muestras tan grandes como las de PISA, confirma la necesidad de reflexionar sobre la forma de hacer los estudios. El propio equipo de PISA, atento a los estudios que se publican al respecto, ya ha publicado por su parte indicaciones sobre cómo realizar este tipo de estudios empíricos.

En esta misma línea, la relativa poca presencia de estudios de corte educativo entre los realizados puede vincularse con una falta de formación de los profesionales e investigadores de la educación en metodologías que permitan abordar este tipo de análisis. El peso de lo local y de lo contextual, fundamental en el ámbito educativo, no puede hacer olvidar la necesidad también de que investigadores con sensibilidad, interés y formación educativa aborden $\mathrm{y}$ participen en las grandes evaluaciones internacionales, $y$ puedan hacer análisis a partir de sus datos.

En todos los documentos revisados se hacen recomendaciones de diferente tipo. La mayor parte de las recomendaciones se centra en medidas políticas, seguido de recomendaciones sobre las futuras investigaciones $\mathrm{y}$ las recomendaciones didácticas. En menor medida estas recomendaciones se centran en cuestiones relacionadas con la metodología de los estudios PISA. Este hecho puede ser debido a la gran dificultad a la hora de interpretación de metodología utilizada en los estudios PISA, el hecho debido a la complejidad de elaboración de cuestionarios y metodología de análisis utilizado, lo cual refuerza la necesidad de una formación metodológica especializada para poder abordar este tipo de análisis.

Desde esta perspectiva, la implicación de equipos de investigadores ajenos a la OCDE, a veces multidisciplinares, frecuentemente de instituciones y países diferentes, puede ayudar a profundizar en toda la información disponible, aportando estudios específicos sobre variables concretas en las que cada equipo puede ser especialista, o estudios comparados, no abordados realmente por
PISA, que permitan extraer conclusiones relevantes respecto a tópicos precisos.

Para ello, desde la OCDE se debe incrementar el esfuerzo por la claridad y disponibilidad de los datos. Pese a los avances realizados al respecto, las bases son poco utilizadas para estudios empíricos, y no siempre de forma adecuada. Medidas complementarias desde el propio equipo PISA pueden ayudar a subsanar estas deficiencias. Por otro lado, desde las instituciones de investigación se debe mostrar la relevancia de que sus investigadores puedan profundizar en estos datos y aportar vías de profundización y análisis. La excesiva simplicidad con que frecuentemente se leen los resultados de PISA, y el gran impacto social de estas lecturas simplistas, deben ser un aliciente fundamental para que a partir de investigación empírica fundamentada se pueda contribuir a hacer una lectura adecuada de este estudio, haciendo contrapeso a lecturas interesadas y en ocasiones sesgadas. Se trata, como finalidad conjunta, de tener una mejor representación de qué ocurre en los sistemas educativos, y cómo se puede contribuir colaborativamente a que la educación sea la mejor posible para todos y todas.

\section{Referencias}

Amador, M. \& López-González, E. (2007). Una aproximación bibliométrica a los modelos multinivel. RELIEVE, 13(1). Disponible en: http://www.uv.es/RELIEVE/v13n1/RELIEV Ev13n1_3.htm

Batista, J. (2016, enero 26). Cómo desmontar el informe PISA. Las Provincias. Disponible en:

http://www.lasprovincias.es/comunitat/2016 01/26/como-desmontar-informe-pisa20160126001834-v.html

Bisquerra, R. (Coord.). (2004). Metodología de Investigación Educativa. Madrid: Muralla.

Bottani, N. (2006). La más bella del reino: el mundo de la educación en alerta con la 
Pereira, Dora; Perales, María-Jesús \& Bakieva, Margarita (2016). Análisis de tendencias en las investigaciones realizadas a partir de los datos del Proyecto PISA. RELIEVE, 22(1), art. M10. DOI: http://dx.doi.org/10.7203/relieve.22.1.8248

llegada de un príncipe encantador. Revista de Educación, extraordinário, 75-90.

Carvalho, L.M. (2009). Governando a Educaçao pelo espelho do perito: Uma análise do PISA como instrumento de regulaçao. Educ. Soc., Campinas, 30(109), 1009-1036.

Coe, R. \& Merino, C. (2003). Magnitud del Efecto: Una guía para investigadores $\mathrm{y}$ usuarios. Revista de psicología de la PUCP, 21(1), 146-177.

Cordero, J., Crespo, E. \& Pedraja, F. (2013). Rendimiento educativo y determinantes según PISA: Una revisión de la literatura en España. Revista de Educación, 362, 273297. doi: http://dx.doi.org/10.4438/1988592X-RE-2011-362-161

De la Orden, A. \& Jornet, J. (2012) La utilidad de las evaluaciones de sistemas educativos: el valor de la consideración del contexto. Bordón, 64(2), 69-88.

Gorur, R. (2014). Towards a Sociology of Measurement in Education Policy. European Educational Research Journal, 13(1), 58-72. doi: http://dx.doi.org/10.2304/eerj.2014.13.1.58

Gallardo-Gil, M., Fernández-Navas, M., Sepúlveda-Ruiz, M.P., Serván, M.J., Yus, R. \& Barquín, J. (2010). PISA y la competencia científica: Un análisis de las pruebas de PISA en el Área de Ciencias. RELIEVE, 16(2). doi: http://dx.doi.org/10.7203/relieve.26.2.4138

Jornet, J. (2012). Dimensiones docentes y cohesión social: reflexiones desde la evaluación. Revista Iberoamericana de Evaluación Educativa, 5(1), 349-362.

Jornet, J. (2013, Septiembre). Asignaturas pendientes en las evaluaciones a gran escala. Ponencia en el simposio Metodología de investigación en ámbitos educativos plurales y diversos, presentada en el XVI Congreso Nacional y II internacional de Modelos de Investigación Educativa de AIDIPE, en Alicante.
Jornet, J. (2016, enero 26). La educación no está tan mal; el informe PISA está sobrevalorado. Levante, el Mercantil Valenciano. Disponible en: http://www.levante-emv.com/comunitatvalenciana/2016/01/26/educacion-malinforme-pisa-sobrevalorado/1370614.html

Martínez Rizo, F. (2006). PISA en América Latina: lecciones a partir de la experiencia de México de 2000 a 2006. Revista de Educación, Número extraordinario, 153166, Disponible en http://www.oei.es/evaluacioneducativa/pisa america_latina_martinez_rizo.pdf

Ozmusul, M. \& Atanur, G. (2013). School Policies and Practices at Upper Secondary Schools in Turkey. According to PISA 2009 Data. Mevlana International Journal of Education, 3(4), 186-199. doi: http://dx.doi.org/10.13054/mije.13.69.3.4

Pey-Yan, L. \& Yi-Chen, H. (2015). Statistical techniques utilized in analyzing PISA and TIMSS data in Science Education from 1996 to 2013: A methodological review. International Journal of Science and Mathematics Education, 13, 1449-1468. doi: http://dx.doi.org/10.1007/s10763-014-9558-5

Rutkowski, D. \& Rutkowski, L. (2013). Measuring Socioeconomic Background in PISA: one size might not fit all. Research in Comparative and International Education, 8(3), 259-278. doi: http://dx.doi.org/10.2304/rcie.2013.8.3.259

Torney-Purta, J. (2013). International LargeScale Assesments: challenges in reporting and potentials for secondary analysis. Research in Comparative and International Education, 8(3), 248-258. doi: http://dx.doi.org/10.2304/rcie.2013.8.3.248

Turner, R. (2006). El Programa Internacional para la Evaluación de los Alumnos (PISA). Una perspectiva general. Revista de Educación, Número extraordinario, 45-74. Disponible en: http://www.revistaeducacion.mec.es/re2006/ re2006.pdf . 
Pereira, Dora; Perales, María-Jesús \& Bakieva, Margarita (2016). Análisis de tendencias en las investigaciones realizadas a partir de los datos del Proyecto PISA. RELIEVE, 22(1), art. M10. DOI: http://dx.doi.org/10.7203/relieve.22.1.8248

\section{NOTAS}

1. Este estudio fue realizado en el marco del proyecto $\mathrm{I}+\mathrm{D}+\mathrm{i}$ denominado Sistema Educativo y Cohesión Social: diseño de un modelo de evaluación de necesidades “SECS/EVALNEC”, con referencia EDU2012-34734 financiado por el Ministerio de Economía y Competitividad y cofinanciado con la ayuda del Fondo Europeo de Desarrollo Regional (FEDER) y las ayudas para grupos de investigación de calidad contrastada "Programa Gerónimo Forteza", asimismo con la ayuda de Universitat de València para el desarrollo de las becas predoctorales “Atracció de Talent”.

2. Este estudio es parte de la tesis doctoral de Dora Pereira, con el tema "Proyecto PISA. Aportaciones de la investigación realizada sobre estudios de contexto"

\section{Authors / Autores}

To know more /

Saber más

Pereira, Dora (doralousadopereira@gmail.com).

Profesora e investigadora del Research in Education and Community Intervention (RECI) del Instituto Piaget (Portugal). Especialista en Evaluación educativa. Su investigación se centra en evaluación de sistemas educativos, psicopedagogía y género. Miembro del grupo de Medición y Evaluación (GEM- UV), Miembro de RIIED y RIMESE.

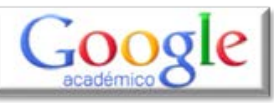

\section{Perales, María-Jesús (perales@uv.es).}

Pedagoga, Doctora en Ciencias de la Educación. Profesora Titular del Departamento de Métodos de Investigación en Educación de la Universidad de Valencia (España). Miembro del Grupo de Evaluación y Medición (GEM-

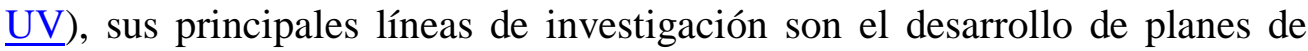
evaluación en ámbitos sociales y de cooperación internacional, y el desarrollo de pruebas de medición para variables socioeducativas. Su dirección postal es: Dpto. MIDE, Avda. Blasco Ibáñez, 30. 46010-Valencia (España)

\section{Bakieva, Margarita (margarita.bakieva@uv.es).}

Máster en Política, Gestión y Dirección de los Centros Educativos y Licenciada en Pedagogía por la Universitat de València. Doctoranda en Educación en la Universidad de Valencia. Miembro del Grupo de Evaluación y Medición (GEM- UV). Desarrolla su investigación en torno al tema de evaluación de la dimensión de la Colegialidad Docente, en el marco de la evaluación de Sistemas Educativos para la Cohesión Social. Su dirección

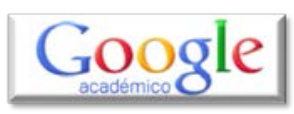
postal es: Dpto. MIDE, Avda. Blasco Ibáñez, 30. 46010-Valencia (España)

\section{RELIEVE}

Revista ELectrónica de Investigación y EValuación Educativa E-Journal of Educational Research, Assessment and Evaluation

[ISSN: 1134-4032]

(C) Copyright, RELIEVE. Reproduction and distribution of this articles it is authorized if the content is no modified and their origin is indicated (RELIEVE Journal, volume, number and electronic address of the document).

(C) Copyright, RELIEVE. Se autoriza la reproducción y distribución de este artículo siempre que no se modifique el contenido y se indique su origen (RELIEVE, volumen, número y dirección electrónica del documento). 